\title{
Spatiotemporal Analysis of Carpooling Data
}

\author{
Gede, $\mathrm{M}^{\mathrm{a}, *}$, Varga, $\mathrm{L}^{\mathrm{a}}$ \\ ${ }^{a}$ ELTE Eötvös Loránd University, Budapest, Hungary, saman@map.elte.hu,vargalola24@gmail.com \\ * Corresponding author
}

Keywords: Carpooling, Spatiotemporal analysis, Transportation mapping

\begin{abstract}
:
Carpooling is a popular alternative to public transport in Hungary. The authors analysed data of the biggest Hungarian carpooling website containing all journeys in a one year interval. The data contains the origin and destination settlements, date/time and price of more than 600000 journeys.

The raw data was first cleaned from typos and misspellings, then geocoded and completed by route geometries using free online services (OpenStreetMap Nominatim and OpenRouteService). Then it was examined from several aspects:

- $\quad$ comparing the average prices and travel times of routes to available public transport means

- $\quad$ analysing the temporal distribution of the journeys through the days of the week, and through the year

- $\quad$ comparing the relative prices of domestic and international journeys

- $\quad$ examining the price deviation of routes - how much does the "free market" standardize fares, and what is the percentage of free rides in various directions
\end{abstract}

The results are visualised on thematic maps and various diagrams.

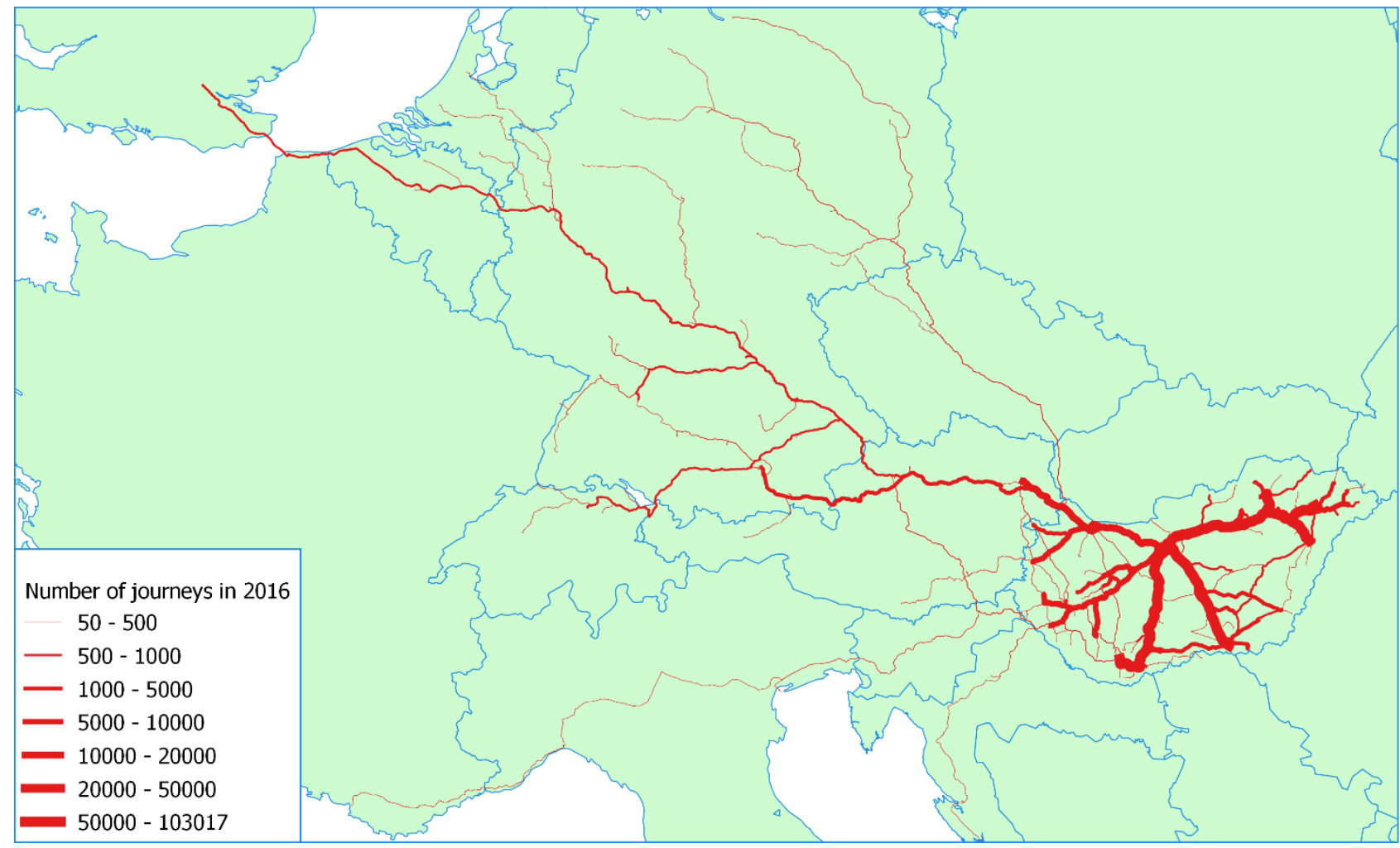

Figure 1. Map of routes with more than 50 journeys in one year

The analysis revealed a great asymmetry between east and west - while the majority of the domestic journeys was realised in the eastern half of Hungary, the international destinations are mostly in Western Europe (Figure 1). The possible causes are that this is where majority of those Hungarians that work can be found, and that traveling from to Romanian cities (which is also very frequent because of the large number of Hungarian minorities there) is mostly realized with a welldeveloped system of minibus companies, so carpooling plays a little role in that direction. 
The temporal distribution is most interesting when examined through the days of the week: the busiest days are Fridays and Sundays, when college students and commuters travel home and back from/to the weekend. The asymmetry between travels from and to Budapest is also observable: on Friday people are leaving the capital for weekend, and on Sunday (and partly on Monday) they are coming back (Figure 2).

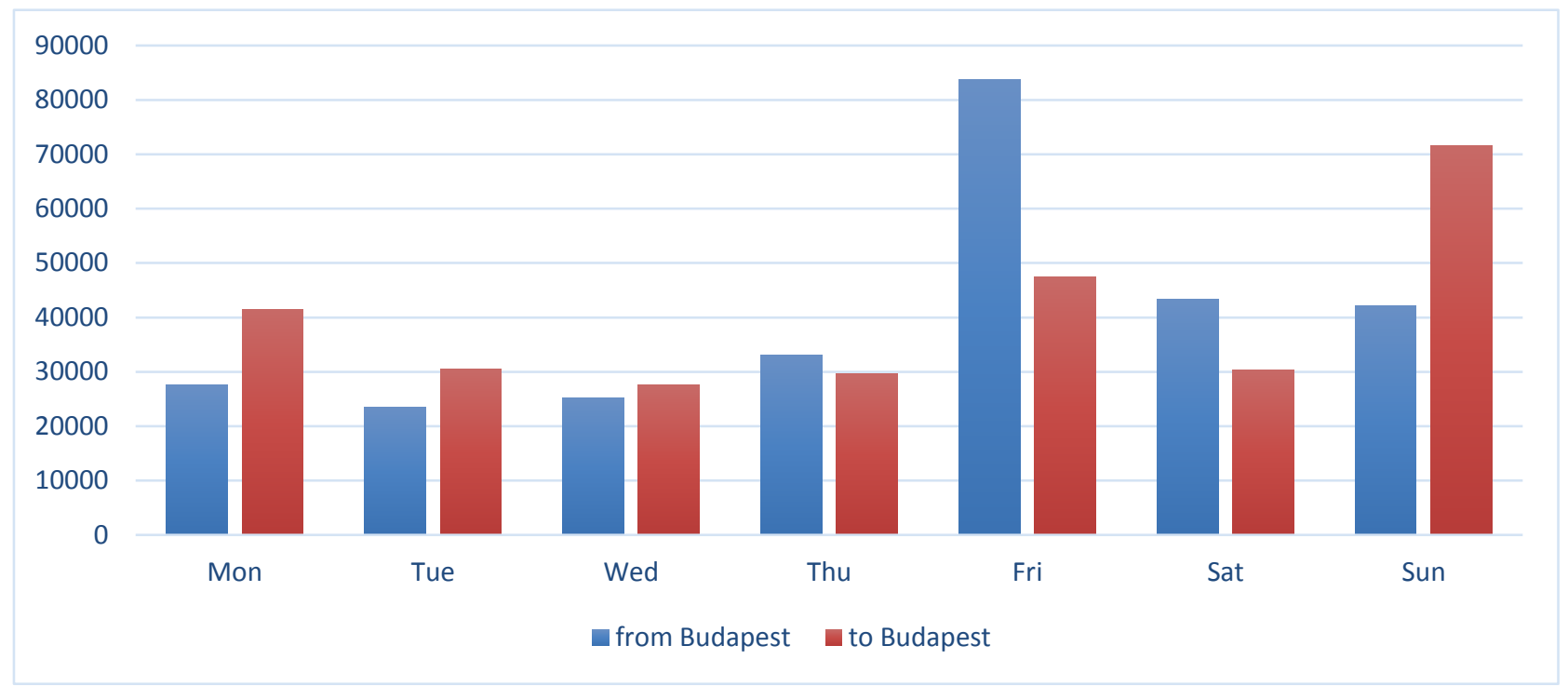

Figure 2. Cumulated number of journeys from/to Budapest on each day of the week 